\title{
Estudos em Reciclagem de Resíduos Plásticos Urbanos para Aplicações Substitutivas de Papel para Escrita e Impressão
}

\author{
Sati Manrich \\ Departamento de Engenharia de Materiais, 3R-nrr, UFSCar
}

\begin{abstract}
Resumo: No presente trabalho, são apresentados os primeiros resultados obtidos em uma série de estudos desenvolvidos com o objetivo principal de obter papel sintético a partir de resíduos plásticos. Diferentes resíduos de polipropileno (PP) foram caracterizados antes e após a formulação de compósitos, alguns dos quais possibilitaram processamentos posteriores por extrusão de filmes para a produção de papéis. Os resultados mostraram a viabilidade técnica do objetivo proposto, tendo sido possível a obtenção de papel para escrita e impressão a partir de resíduos de garrafas de PP, coletados seletivamente em restaurante. Comparativamente ao papel celulósico, apesar de menores propriedades de rigidez, opacidade e de impressão com tintas aquosas principalmente, os filmes de compósitos de PP apresentaram várias características semelhantes, motivando a continuidade dos estudos para o aprimoramento do material.
\end{abstract}

Palavras-chave: Papel sintético, papel plástico, reciclagem, plásticos pós-consumo, resíduos plásticos urbanos, $P P$, compósitos, filmes.

\section{Studies on Recycling of Post-Consumer Plastics Waste for Substitutive Applications in Writable and Printable Paper}

Abstract: In this article, preliminary results obtained in a series of studies performed with the main objective of producing synthetic paper from plastics waste are presented. Different polypropylene (PP) residues were characterized before and after "compounding" with filler and additives. Some of these composites were adequate for film extrusion, which were then used to produce paper. The characterization of composite films showed the technical viability of the proposed objective: a writable and printable plastic composite paper obtained using PP bottles from urban residues. Despite minor reduction in properties related to rigidity, opacity and printing mainly with aqueous ink PP paper showed many similar characteristics, compared to cellulosic paper, that have motivated the continuity of the studies for improving the substitute material.

Keywords: Synthetic paper, plastic composite paper, recycling, post-consumer residues, urban plastics waste, $P P$, composites, films.

\section{Introdução}

A agressão ao meio ambiente causada pelos resíduos originados nos processos, serviços e produtos utilizados na vida moderna tem-se tornado uma preocupação crescente em todos os setores. Os polímeros sintéticos e os naturais modificados, muito utilizados em embalagens diversas, têm sido considerados um dos grandes vilões da poluição ambiental, principalmente quando se refere aos danos causados pelos resíduos urbanos ${ }^{[1,3]}$. A contribuição desses materiais no crescente volume de resíduos sólidos urbanos também tem aumentado ao longo dos últimos anos, tendo alcançado o segundo lugar em maior incidência na composição do lixo da cidade de São Paulo, considerando somente os plásticos ${ }^{[1]}$.

Autor para correspondência: Sati Manrich, Departamento de Engenharia de Materiais, Universidade Federal de São Carlos, CEP: 13565-905, São Carlos, SP. E-mail: sati@power.ufscar.br 
Por outro lado, a derrubada de árvores em grande escala, seja para a produção de papéis, para a fabricação de móveis, como também para a utilização nas construções civis e a geração de energia, requer um controle mais rigoroso de forma a minimizar o impacto ambiental do desmatamento ${ }^{[4,6]}$. Ainda, segundo um estudo feito pelo BNDES em 1996, a produção de papel no país necessitaria ser duplicada até o ano 2005, projetando um investimento da ordem de US $\$ 4,8$ bilhões ${ }^{[4]}$. Estudos mais recentes ${ }^{[5]}$ mostram que, caso a capacidade de produção do setor seja mantida nos mesmos níveis atuais, o Brasil passará de exportador a importador de papel, já em 2001.

$\mathrm{O}$ aumento da produção de papel, nos níveis previstos, tem um fator complicador, uma vez que vai depender essencialmente da disponibilidade de celulose, cuja matéria prima básica é a madeira ${ }^{[4,6]}$. No Brasil, essa matéria prima é exclusiva de reflorestamentos próprios, cuja área total precisará de uma expansão significativa até o ano 2005, necessitando para isso investimentos de US\$ 930 milhões. Sem contar que as florestas a serem cultivadas nas áreas de expansão começarão a dar retorno somente após um período superior a sete anos ${ }^{[4,5]}$.

Estima-se também que a oferta mundial de papel não irá se expandir no mesmo ritmo do crescimento econômico, sendo calculada em 320 milhões de t/ano, com um déficit significativo de 70 milhões t para o ano de 2005, sendo o segmento de papel para escrita e impressão o que apresentará a maior defasagem: 27 milhões de toneladas ${ }^{[4,5]}$.

A fabricação de papel sintético ou papel plástico, a partir de termoplásticos é apontada como uma das alternativas mais viáveis e promissoras para o preenchimento dessa lacuna de produção do papel celulósico ${ }^{[6,7]}$. A possibilidade de estar inovando com uma única alternativa para duas problemáticas, motivou o início das pesquisas em papel sintético, utilizando para isso matéria prima reciclada de resíduo urbano, ou seja, plástico proveniente de lixo ${ }^{[8,9]}$.

O papel plástico pode ser competitivo em termos de custo como também apresenta vantagens com relação à utilização de tecnologias relativamente limpas para a sua fabricação, quando comparado ao papel celulósico. Em casos específicos, a relação custo/benefício pode justificar a utilização do papel sintético, a exemplo dos recentes investimentos do governo brasileiro em optar pela fabricação de notas de dez Reais comemorativas dos 500 anos do descobrimento, com papel plástico e não celulósico. Além disso, com o uso do papel sintético, existe a possibilidade da confecção de livros, revistas, cadernos escolares, jornais, papéis para escritório, além de produtos monocomponentes (por exemplo, garrafas, tampas e rótulos de papel de polipropileno), facilitando vantajosamente o processo de reciclagem pós-consumo. Este processo, por sua vez, viabiliza o fechamento do ciclo de vida do produto, tornando-o ambientalmente correto ${ }^{[10]}$.

Na maioria dos desenvolvimentos e pesquisas sobre papel sintético, cujas publicações são bastante raras, o objetivo principal tem sido o de reproduzir as propriedades do papel celulósico em filmes plásticos, por meio da formação de microcavitações durante o processo de orientação ${ }^{[11-14]}$. A cavitação provocada por esse processo é o que confere, basicamente, a opacidade, a receptividade a tintas e a baixa densidade necessária $S^{[-8,8-12}$. Tem-se conseguido algum sucesso com filmes de compósitos de poliolefinas-carga inorgânica, blendas de polímeros incompatíveis ou mesmo por meio de uma extensiva orientação, abaixo da temperatura de fusão, de polímeros semi-cristalinos. Nesses casos, as microfases constituídas ou por partículas de carga, ou por partículas dispersas de polímero incompatível, ou ainda por partículas de fase cristalina, servirão de núcleos para a formação das microcavitações.

Foi proposto também a mistura de fibras e/ou microfibras de poliolefinas ou de poliésteres com as de celulose ${ }^{[16]}$. Dentre os vários métodos, entretanto, os que têm tido melhores resultados são os filmes de compósitos de polipropileno (PP) e polietilenos (PE) com carga inorgânica e os filmes obtidos da orientação de blendas de polímeros semi-cristalinos como o poli(tereftalato de etileno) $(\mathrm{PET})^{[6-8,12-15]}$.

Os estudos apresentados neste trabalho foram realizados em papel sintético feito de compósitos de PP, de diferentes origens com cargas inorgânicas. Em publicações futuras, serão apresentados os resultados obtidos com filmes de blendas de PP/poliestireno (PS) e de PET/poliolefinas.

\section{Experimental}

\section{Materiais}

PP virgem - PP KF-6100 (PPvir) grade específico para a confecção de filmes, doado pela Polibrasil S.A., sem qualquer processamento anterior. 
PP reciclado industrial (PPrec. ind.) - este material, doado pela Koppol Films, teve uma seqüência de processamentos: (a) extrusão de filmes biorientados de PP KF 6100; (b) filmes que foram rejeitados na inspeção de qualidade foram picotados, aglutinados, extrudados e finalmente granulados.

PP de resíduo urbano (PPres. urb.): algumas caracterizações inicialmente foram feitas com diferentes produtos de PP presentes como resíduo urbano: garrafas e copos de água mineral, que foram retirados de uma coleta seletiva em restaurante. Para a confecção dos filmes, decidiu-se utilizar apenas as garrafas de água mineral, conforme comentários posteriores.

Carbonato de Cálcio - doado pela Polibrasil S.A. e pela empresa Química Barra do Piraí, de diferentes granulometrias. Aditivos: $\mathrm{TiO}_{2}$, forma rutilo, doado pela Polibrasil S.A. e antioxidante Irganox B215, doado pela Ciba.

\section{Métodos}

As garrafas de água mineral pós-consumo foram lavadas com água ou em conjunto com detergente quando necessário, secadas ao ar e então moídas num moinho de facas. O material moído na forma de flakes foi peneirado para a separação dos pós, os quais poderiam provocar uma degradação maior nos processamentos posteriores. Este material foi utilizado diretamente na obtenção dos compósitos.

\section{Caracterização da Matéria-Prima}

A caracterização do comportamento reológico de 3 tipos de PP (virgem, reciclado industrial e de garrafas de água) foi feita utilizando um Reômetro Capilar Instron, modelo 321l, a $200{ }^{\circ} \mathrm{C}$. A realização dos testes de índice de fluidez baseou-se na norma ASTM D1238. Para a caracterização do comportamento térmico, as amostras moídas de diferentes tipos de PP foram todas submetidas a um mesmo tratamento térmico. Essas amostras foram então analisadas por meio de Calorimetria Diferencial de Varredura (DSC), Calorímetro DuPont Modelo 2910; o ensaio foi realizado sob uma taxa de aquecimento/ resfriamento de $10{ }^{\circ} \mathrm{C} / \mathrm{min}$. O ensaio de tempo de indução oxidativa (OIT) teve como base a norma ASTM D-3895, com modificações feitas pela ABNT e Telebrás. O Calorímetro DuPont 2910 foi também utilizado para esse ensaio.

A carga foi caracterizada somente com relação à granulometria do carbonato de cálcio, proveniente de diferentes origens: Polibrasil Resinas S.A (PBR) e Química Barra do Piraí (QBP). O objetivo foi determinar a influência da granulometria da carga sobre as propriedades do papel sintético. Essa determinação foi feita em Sedígrafo, Micromeritics $5000 \mathrm{D}$, o qual se baseia na lei de Stokes de sedimentação, utilizando um detector de raio-X.

\section{Formulação e Caracterização de Compósitos}

A formulação dos compósitos foi baseada em trabalho da literatura, principalmente com relação à Patente de Ohno/OJI YUKA CO. Ltda. ${ }^{[13]}$. A primeira formulação consistiu de $80 \%$ de PP virgem e $20 \%$ de $\mathrm{CaCO}_{3} \mathrm{PBR}$, proporção em peso, com uma adição posterior de $0,3 \%$ em peso de $\mathrm{TiO}_{2}$.

A segunda e terceira formulações foram feitas com $60 \%$ PPrec. ind. $+40 \% \mathrm{CaCO}_{3}$ PBR e $60 \%$ PPres.urb. $+40 \% \mathrm{CaCO}_{3} \mathrm{PBR}$, respectivamente, ambas com adições posteriores de $0,3 \%$ de $\mathrm{TiO}_{2}$. Na quarta formulação, comparada à terceira, variou-se apenas a granulometria da carga, isto é, de $\mathrm{CaCO}_{3} \mathrm{PBR}$ para $\mathrm{CaCO}_{3}$ QBP. Foi feita uma quinta formulação a partir desta quarta, adicionando-se PP virgem KF 6100, em que a proporção de carga foi diminuída novamente para $20 \%$.

A mistura das cargas inorgânicas foi feita em extrusora rosca-dupla da Werner-Pfleiderer $(30 \mathrm{~mm})$. As seguintes condições de processamento foram utilizadas: velocidade de rotação do parafuso $190 \mathrm{rpm}$, com um perfil de temperaturas dividido em 6 zonas: $188,198,220,243,240$ e $220^{\circ} \mathrm{C}$. A carga foi adicionada após a zona de alimentação da resina. Obtidos os compósitos, os respectivos índices de fluidez (MFI) foram determinados.

\section{Obtenção e Caracterização de Pape/ Sintético}

Para a obtenção de papel sintético, foi utilizada uma extrusora sopro de filmes (film blowing), CIOLA IF50, cujo perfil de temperaturas foi de 180, 200 e $230{ }^{\circ} \mathrm{C}$. Para cada formulação, as condições de vazão do ar comprimido para inflar o tubo foram inicialmente ajustadas, até se conseguir um filme homogêneo. A partir de então, a velocidade de puxamento do filme foi ajustada para $0,8 \mathrm{~m} / \mathrm{s}$ de forma a obter a espessura desejada.

A resistência ao rasgamento foi determinada com base na norma ASTM D1004; a caracterização da deformação permanente sob calor (DPC), específica para folhas plásticas (filmes), materiais plásticos 
laminados e plásticos moldados, foi baseada na norma ASTM D794. A caracterização da opacidade foi realizada em equipamento da Polibrasil Resinas S.A., Haze Gardplus. A densidade foi calculada determinandose a massa e a espessura de amostras cortadas nas dimensões de $5 \times 5 \mathrm{~cm}^{2}$. Essas determinações foram feitas em substituição à "gramatura" (massa por unidade de área), uma vez que as espessuras entre os diferentes tipos de papel variaram significativamente, não permitindo tal comparação.

A caracterização microscópica em termos de dispersão das cargas e formação de microcavitações foi feita por meio de Microscopia Eletrônica de Varredura (SEM), com microanálise (EDS), em microscópio OXFORD modelo eXL.

Verificou-se qualitativamente a possibilidade de diversos tipos de escrita (lápis, caneta esferográfica, tinteiro e hidrográfica) e de impressão (matricial, a laser e por jato de tinta) sobre os papéis produzidos.

\section{Resultados e Discussão}

\section{Caracterização da Matéria Prima}

Os valores das propriedades determinadas por DSC e por plastômetro, para os diferentes tipos de PP, tais como temperatura de fusão $\left(\mathrm{T}_{\mathrm{m}}\right)$ e grau de cristalinidade $\left(\mathrm{x}_{\mathrm{c}}\right)$, do tempo de indução oxidativa (OIT) e do índice de fluidez (MFI), já foram apresentados anteriormente ${ }^{[17]}$. Assim, esses resultados serão apenas comentados aqui e não serão listados na forma de tabelas. Resíduos de PP originários de copos de água mineral também foram caracterizados, quanto à $\mathrm{T}_{\mathrm{m}}, \mathrm{x}_{\mathrm{c}}$ e OIT.

De forma geral, os resultados de $T_{m}, x_{c}$ e OIT obtidos para amostras de PP de diferentes origens mostraram valores relativamente próximos dos valores do PP virgem e dos resíduos de copos de água. Entretanto, as caracterizações desta última fonte de matéria prima não tiveram continuidade devido às dificuldades em sua utilização, resultantes da presença de tintas de impressão, que poderiam se volatilizar durante o reprocessamento, e a necessidade de incluir mais uma etapa, a de aglutinação, para sua recuperação.

Os resultados de MFI mostraram que o valor para o PP de resíduo de garrafa de água é mais baixo, porém não difere muito daquele do $\mathrm{PP}$ virgem $(3,28$ e 4,11 g/10 min, respectivamente). Neste caso, os materiais correspondem a grades específicos para as respectivas aplicações. Por outro lado, o valor de MFI do material reciclado industrial é mais de 6 vezes o do PPvir. Neste caso, por serem ambos os polímeros originários de mesmo grade (PP KF 6100), o MFI mais alto do PPrec. ind. (26,7 g/10 min) estaria confirmando que os vários reprocessamentos provocaram uma degradação mais intensa, com diminuição de peso molecular. No ensaio de OIT, novamente, o menor valor para o PPrec.ind veio a confirmar estar este material em um estágio mais avançado de degradação do que os demais.

Os resultados obtidos na caracterização reológica realizada para três tipos de PP, virgem, reciclado industrial e resíduo urbano (garrafa de água mineral), também já foram publicados ${ }^{[17]}$. Observou-se que a viscosidade da amostra de PPres.urb. é a que apresentou o maior valor, apesar de ter sido retirada de material pós-consumo. Conforme já comentado, essas diferenças são devido principalmente ao fato do grade de polipropileno utilizado ser específico para garrafas, ao contrário dos dois outros casos, PPvir e PPrec.ind, os quais são específicos para filmes. Observou-se também que os valores de viscosidade apresentados pelo PPres.urb. não são tão mais altos em comparação com os de PPvir, principalmente sob taxas altas de cisalhamento.

Os valores do índice $\mathrm{n}$, determinados por meio da lei das potências: $\mathrm{n}=0,33$ para o PPvir, $\mathrm{n}=0,46$ para $\mathrm{o}$ PPrec.ind e $n=0,31$ para o PP de resíduo urbano. Neste caso, como o índice n do PPres.urb. foi o de menor valor $(n=0,31)$, este material irá apresentar uma diminuição maior na viscosidade para um mesmo aumento de $\gamma$, comparado aos demais. Este valor de $\mathrm{n}$, no entanto, é muito próximo daquele do PP virgem $(\mathrm{n}=0,33)$. Isso confirma a possibilidade de se utilizar os mesmos equipamentos e as mesmas condições para a obtenção do filme de PP de resíduo urbano.

Com o objetivo de verificar o efeito da variação na granulometria da carga $\mathrm{CaCO}_{3}$, principalmente nas propriedades óticas e de receptividade a tintas (formação de microcavitações), resolveu-se utilizar uma carga com granulometria menor. A Tabela 1 apresenta os resultados da análise granulométrica para os dois tipos de carga.

A Tabela 1 mostra uma diferença significativa no tamanho das partículas entre os dois tipos de carbonato. Mais de $80 \%$ das partículas do $\mathrm{CaCO}_{3} \mathrm{QBP}$ apresentam tamanho menor do que os $20 \%$ menores do $\mathrm{CaCO}_{3} \mathrm{PBR}$. Conforme será comentado posteriormente, essa diferença teve uma influência significativa nas características dos compósitos e na fabricação do papel. 
Tabela 1. Distribuição granulométrica das partículas de carga $\mathrm{CaCO}_{3}$

\begin{tabular}{cccc}
\hline Origem: Polibrasil Resinas (PBR) & \multicolumn{2}{c}{ Origem: Química Barra do Piraí (QBP) } \\
\hline $\begin{array}{c}\text { Diâmetro esférico } \\
\text { equivalente } \\
(\mu \mathbf{m})\end{array}$ & $\begin{array}{c}\text { Porcentagem em massa } \\
\text { cumulativa } \\
(\%)\end{array}$ & $\begin{array}{c}\text { Diâmetro esférico } \\
\text { equivalente } \\
(\mu \mathbf{m})\end{array}$ & $\begin{array}{c}\text { Porcentagem em massa } \\
\text { cumulativa } \\
(\%)\end{array}$ \\
\hline 2,00 & 6,00 & 0,76 & 6,00 \\
4,10 & 20,0 & 1,15 & 20,0 \\
6,90 & 40,0 & 1,60 & 40,0 \\
10,4 & 60,0 & 2,30 & 60,0 \\
14,5 & 80,0 & 3,70 & 80,0 \\
18,0 & 90,0 & 5,15 & 90,0 \\
21,0 & 95,0 & 7,40 & 95,0 \\
\hline
\end{tabular}

Os valores de índice de fluidez obtidos após a formulação dos compósitos de PP com carga e aditivos foram: 2,$78 ; 9,17 ; 1,26$ e $1,10 \mathrm{~g} / 10 \mathrm{~min}$ para PPvir/ $20 \% \mathrm{CaCO}_{3}$ PBR; PPrec.ind. $40 \% \mathrm{CaCO}_{3} \mathrm{PBR}$; PPres.urb. $/ 40 \% \mathrm{CaCO}_{3}$ PBR e PPres.urb. $/ 40 \% \mathrm{CaCO}_{3}$ QBP, respectivamente

Com esses dados verificou-se que, possivelmente, o compósito de PPvir não apresentaria dificuldades na obtenção de filmes soprados, uma vez que a sua fluidez (2,78 g/10 min) não diminuiu acentuadamente comparada com a do PP virgem sem carga $(4,11 \mathrm{~g} / 10$ min). $\mathrm{O}$ índice de fluidez relativamente baixo para ambos os compósitos de PPres.urb. e muito elevado para o compósito PPrec.ind $/ \mathrm{CaCO}_{3}$ poderiam acarretar em dificuldades na obtenção do filme, provocando pressões maiores ou rupturas do filme tubular.

Conforme previsto, o processo de extrusão de filme soprado para o compósito de PPvir não apresentou qualquer dificuldade. Foram utilizadas as condições usuais para o processamento do PP virgem puro. Ao longo do processamento, foram adicionados concentrados de $\mathrm{TiO}_{2}$, de forma que a sua proporção chegou a triplicar, isto é, passou de $0,3 \%$ para $1,0 \%$. Quando este processamento foi aplicado ao PP reciclado industrial surgiram problemas, e não foi possível a obtenção de um filme homogêneo. Além disso, o filme tubular (bolha) rompeu relativamente rápido, de forma que não se conseguiu grande quantidade de amostra do papel sintético de PPrec.ind. Para o PPres.urb./ $\mathrm{CaCO}_{3} \mathrm{PBR}$, por outro lado, apesar do baixo valor do índice de fluidez, o seu processamento ocorreu nas mesmas condições do compósito de PPvir sem problemas.
Já a quarta formulação constituída por PP res.urb./ $\mathrm{CaCO}_{3}$ QBP, apesar de ter sido tratada nas mesmas condições da terceira formulação e ter apresentado um índice de fluidez bastante próximo daquela, não foi possível a obtenção de papel a partir dela. No momento da formação da "bolha" a partir da matriz da extrusora, sequer formou um filme tubular. As partículas muito finas do carbonato da Química Barra do Piraí formaram aglomerados, impedindo uma dispersão homogênea do pó. Assim, apesar do índice de fluidez ter sido próximo ao do compósito anterior, os aglomerados não permitiram a formação de um filme contínuo de PP.

\section{Caracterização do Pape/ Sintético}

$\mathrm{Na}$ Tabela 2 estão listados os resultados de densidade e de tensão máxima para início de rasgamento, para amostras de diferentes tipos de papel. Esta última determinação foi feita em duas direções quando possível, longitudinal e transversal à da extrusão, para os filmes de PP e em uma única direção para diferentes tipos de papel celulósico.

A densidade mais alta foi apresentada pelo filme de PPvir, apesar da proporção de $\mathrm{CaCO}_{3}$ ter sido a metade da usada nos demais. O papel de PPrec.ind apresentou a densidade mais baixa, provavelmente devido à presença de muitos vazios. Esses vazios não se constituíram de microcavitações, que seriam desejáveis, conforme será verificado posteriormente. O papel de PPres.urb. (terceira formulação) era de um filme homogêneo, sem vazios ou "buracos", que levariam à obtenção de uma densidade mais baixa. Apesar de a densidade ser bem próxima da de um 
Tabela 2. Valores de densidade e de tensão máxima para início de rasgamento de diferentes tipos de papel.

\begin{tabular}{|c|c|c|c|c|c|}
\hline Papel & $\begin{array}{l}\text { Espessura } \\
\qquad(\mu \mathbf{m})\end{array}$ & $\begin{array}{c}\text { Densidade } \\
\left(\mathrm{g} / \mathrm{cm}^{3}\right)\end{array}$ & Direção & $\begin{array}{l}\text { Espessura } \\
\qquad(\mu \mathbf{m})\end{array}$ & $\begin{array}{c}\text { Tensão Máxima } \\
\text { (N) }\end{array}$ \\
\hline \multirow{2}{*}{ PPvir } & \multirow{2}{*}{63,3} & \multirow{2}{*}{0,84} & Longitudinal & 66,1 & 7,45 \\
\hline & & & Transversal & 64,5 & 7,26 \\
\hline PPrec.ind. & 68,3 & 0,72 & $\begin{array}{l}\text { Longitudinal } \\
-\end{array}$ & 73,0 & 5,76 \\
\hline \multirow{2}{*}{ PPres.urb. } & \multirow{2}{*}{67,0} & \multirow{2}{*}{0,82} & Longitudinal & 67,1 & 4,88 \\
\hline & & & Transversal & 65,0 & 4,00 \\
\hline Celulósico (caderno) & 66,7 & 0,74 & - & 84,0 & 11,50 \\
\hline \multirow{2}{*}{ Celulósico (sulfite) } & \multirow{2}{*}{97,3} & \multirow{2}{*}{0,79} & Tipol & 112,0 & 21,20 \\
\hline & & & Tipo2 & 121,0 & 19,80 \\
\hline
\end{tabular}

papel sulfite (celulósico), a rigidez do papel de PPres.urb., do mesmo modo que os demais papéis sintéticos, é menor.

Verifica-se pela Tabela 2 que as espessuras dos filmes sintéticos são na maioria dos casos menores do que as dos celulósicos, e as respectivas resistências ao rasgamento também. A menor espessura foi obtida com o PP de resíduo urbano (garrafa de água mineral). Conforme já comentado anteriormente, a rigidez do papel de PPres.urb. é bem baixa também. Os filmes mais finos, entretanto, não justificam essa diferença significativa nas propriedades mecânicas do papel sintético e celulósico. É possível que a norma utilizada para o ensaio de rasgamento, sendo específica para filmes plásticos, não tenha sido adequada para essa comparação.

A deformação permanente sob calor (DPC) e a opacidade para os três diferentes tipos de papel sintético são dadas na Tabela 3. A DPC foi avaliada em termos da variação no aspecto, cor e variações na massa e dimensão das amostras e a opacidade, em termos de porcentagem de luz transmitida (\%T).

Apesar de ter observado perda de massa em proporções muito baixas, no ensaio de DPC, não foi possível detectar qualquer variação nas dimensões dos diferentes tipos de papel. Não foi observado também qualquer alteração na cor e no aspecto, nem mesmo um encolhimento provocando enrugamento, o que poderia ser esperado. Na prática, verificou-se que a opacidade não é muito boa para os filmes de PPrec.ind e Pres.urb., porém o aspecto é semelhante ao papel natural. A opacidade do filme de PPvir é comparável à do papel celulósico, apesar da diferença nos valores da Tabela 3, porém este filme apresentou brilho, com um aspecto muito próximo de plástico.

As micrografias apresentadas na Figura 1 mostram a superfície do papel de PPrec.ind. e PPres.urb., onde se confirma uma boa dispersão das partículas de carga nos dois casos e a presença de vazios em grande proporção, no primeiro caso. É possível verificar também que as partículas de carga, na superfície, ficam recobertas por uma fina camada de polipropileno.

Os resultados da microanálise EDS feita em PPrec.ind. e PPres.urb. mostraram outros tipos de partículas normalmente presentes em uma composição típica de $\mathrm{CaCO}_{3}: \mathrm{MgCO}_{3}$ e $\mathrm{SiO}_{2}$. Novamente se observa uma distribuição bastante homogênea, com algumas regiões de concentração associadas às

Tabela 3. Resultados do teste de deformação permanente sob calor (DPC) e de opacidade.

\begin{tabular}{lccc}
\hline \multicolumn{1}{c}{ Papel } & $\begin{array}{c}\text { DPC dimensões } \\
(\mathbf{\%})\end{array}$ & $\begin{array}{c}\text { DPC em massa } \\
(\mathbf{\%})\end{array}$ & $\begin{array}{c}\text { Opacidade } \\
(\mathbf{\% T})\end{array}$ \\
\hline PPvir & 0 & 0,18 & 48,0 \\
PPrec.ind & 0 & 0,25 & 80,0 \\
PPres.urb. & 0 & 0,22 & 61,0 \\
Celulósico (sulfite) & - & - & 20,1 \\
\hline
\end{tabular}




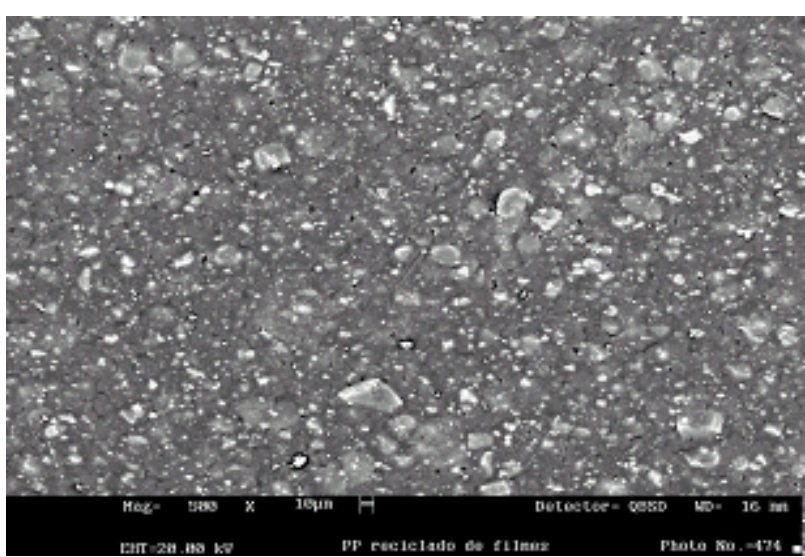

(a)

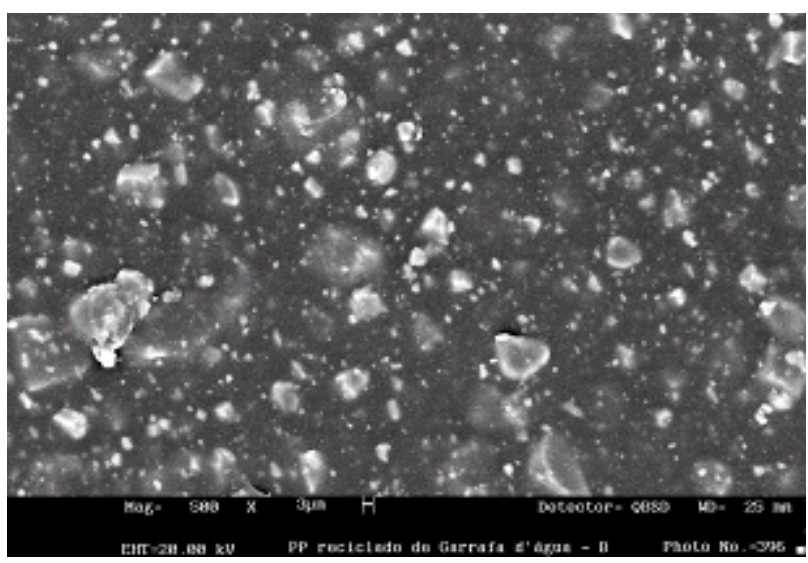

(b)

Figura 1. Micrografia da superfície de dois papéis sintéticos: (a) PP reciclado industrial e (b) PP de resíduo urbano (garrafa de água mineral).

partículas ou aglomerados de partículas. Tendo-se determinado a granulometria da carga, foi possível confirmar que essas regiões de concentração são devidas às partículas maiores. Isto pode ser verificado comparando-se os dados da Tabela 1 e Figura 1 com a Figura 2(a), onde se mostra o mapeamento de papel PPres.urb. em termos do elemento Cálcio.

A varredura feita com os elementos Magnésio, Silício e Titânio confirmou uma distribuição homogênea da carga e do pigmento, conforme pode ser observado na Figura 2(b) para o pigmento branco de Ti. Verifica-se que o mapeamento desse pigmento, que não faz parte da composição de $\mathrm{CaCO}_{3}$, apresenta um distribuição totalmente homogênea e sem regiões de concentração, ao contrário dos elementos $\mathrm{Mg}$ e Si, que se concentraram nas mesmas posições do Cálcio, apesar de em proporções menores.

Os vazios no PPrec. ind. foram considerados "buracos" ou "furos", uma vez que apenas aqueles com 50 a 1.500 A $(0,0050$ a $0,15 \mathrm{~mm})$ são

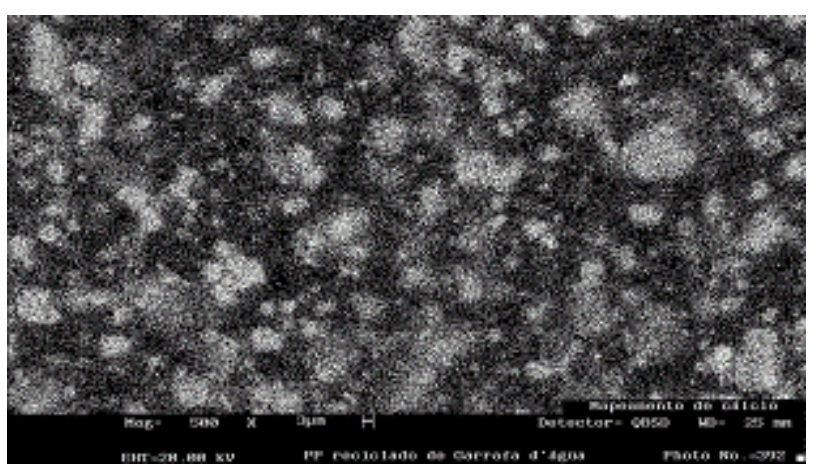

(a)

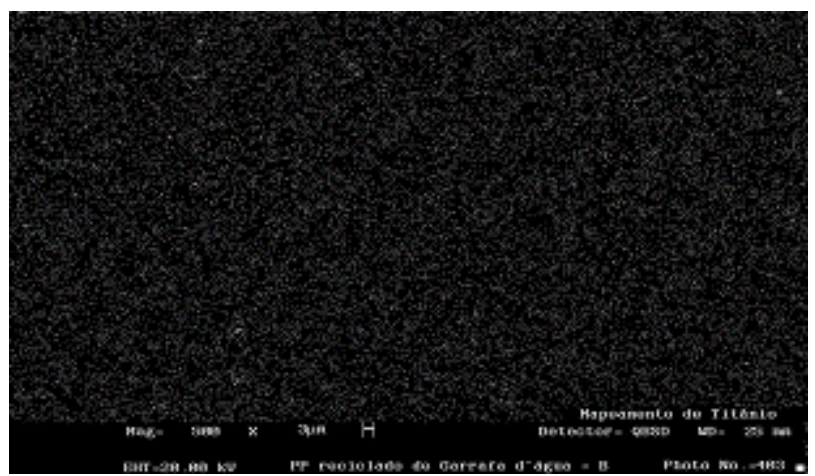

(b)

Figura 2. Microanálise por EDS no papel de PPres.urb.: (a) $\mathrm{Ca}\left(\mathrm{CaCO}_{3}\right)$, (b) $\mathrm{Ti}\left(\mathrm{TiO}_{2}\right)$.

considerados microcavitações. Os vazios observados nesse papel, por outro lado, medem 3 a $5 \mu \mathrm{m}$, em média. Além disso, as microcavitações formam-se na interface polímero/partículas de carga, como uma conseqüência da orientação ao estirar o filme. $\mathrm{Na}$ Figura 3(a), é nítido que os vazios se formam na matriz polimérica.

A Figura 3(b), mostra o que pode ser considerado como microcavitações e que foram encontrados no papel de PPres.urb. Os vazios chegam a ter no máximo $0,4 \mu \mathrm{m}$ e se formam na interface polímeropartícula de carga. Estes vazios, porém, apareceram em quantidades desprezíveis, pelo menos na superfície.

A Figura 4 mostra uma micrografia da superfície de papel de PPres.urb., onde foi realizado um teste de escrita com grafite. Apesar dessa micrografia apresentar uma imagem em que o grafite parece estar totalmente solto na superfície, este papel apresentou uma boa receptividade e retenção do grafite. O mesmo ocorreu com o papel de reciclado industrial, ao contrário do de PP virgem.

Para os três tipos de papel sintético, foram realizados testes com diferentes formas de escrita $\mathrm{e}$ 


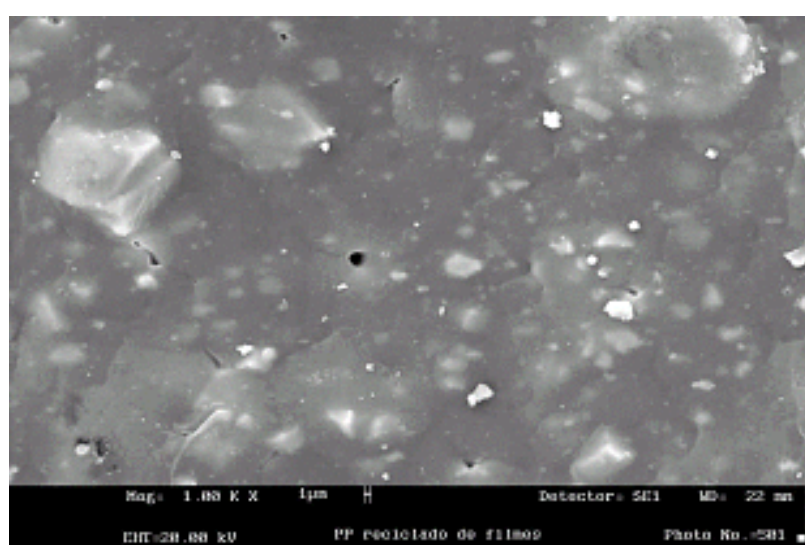

(a)

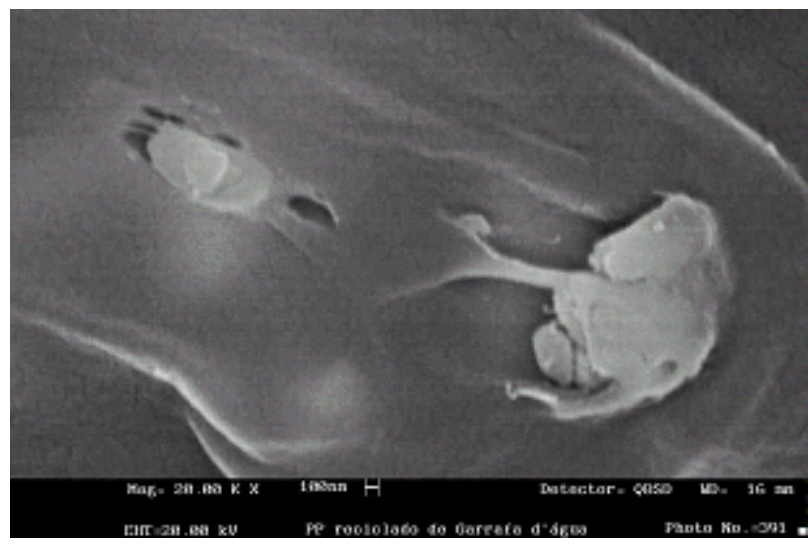

(a)

Figura 3. Vazios na superfície do papel sintético: (a) buracos no PPrec.ind e (b) microcavitações no PPres.urb.

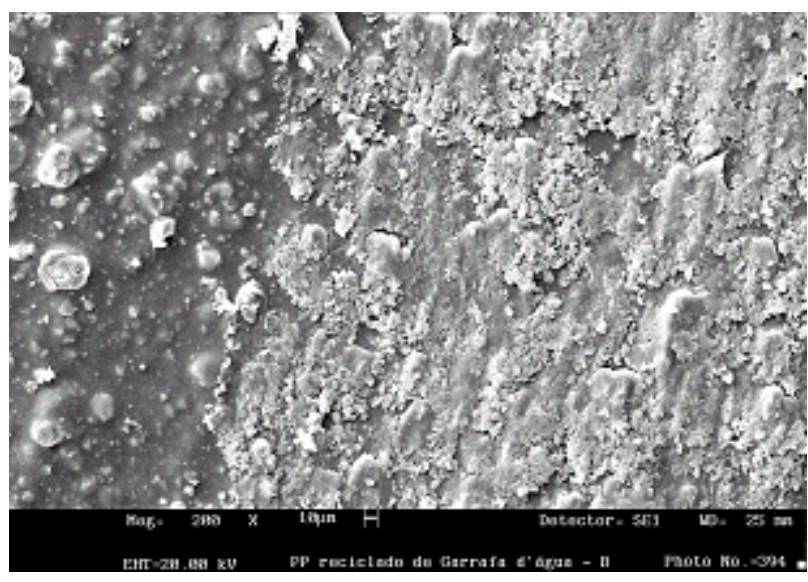

Figura 4. Grafite sobre a superfície do papel de PPres.urb.

impressão: grafite, caneta esferográfica, tinteiro e hidrográfica, e impressoras matricial, jato de tinta e laser. Para os papéis de PPres.urb. e PPrec.ind., foi possível a escrita com grafite e caneta esferográfica, impressão matricial e , após tratamento corona, impressão com jato de tinta apenas para o de resíduo pós-consumo. Para o papel de PPvir, não foi possível obter escrita ou impressão de qualidade, mesmo que razoável.

Os resultados obtidos neste primeiro estudo motivou a sua continuidade, principalmente com relação ao desenvolvimento de inovações tecnológicas, utilizando outros materiais e processos alternativos, visando o melhoramento das propriedades mecânicas e de superfície, com a formação de microcavitações e aumento da energia superficial. Esses resultados deverão ser publicados no futuro.

\section{Conclusões}

- Entre os três tipos de PP utilizados como matéria prima para a confecção do papel sintético, o reciclado industrial apresentou um estágio mais avançado de degradação, o que resultou em altíssimo índice de fluidez. Isso provocou dificuldades no processo de extrusão sopro de filmes, levando à obtenção de um filme com muitos vazios na forma de furos.

- Este trabalho mostrou ser viável reciclar garrafas sopradas de PP pós-consumo por meio de extrusão de filmes de compósitos altamente carregados, para obtenção de papel sintético com várias características de papel celulósico.

- As microcavitações necessárias para a receptividade a tintas praticamente não se formaram na superficie do papel sintético. Isto não impediu, entretanto, que os papéis de PPrec.ind. e de PPres.urb. apresentassem receptividade e retenção a alguns tipos de escrita: grafite e esferográfica. Para o PPres.urb., foi possível também a impressão matricial e jato de tinta preta, porém não é compatível com tintas ou adesivos à base de água, devido à natureza apolar da matriz polimérica.

- Os resultados bastante positivos da presente pesquisa indicam ser viável a fabricação de papel sintético para substituição do papel celulósico, a partir do reaproveitamento de resíduos plásticos urbanos. Vários aprimoramentos, no entanto, estão sendo feitos como aumento da rigidez aliado a uma diminuição na densidade, bem como ao aumento na opacidade e receptividade a outros tipos de escrita e impressão.

\section{Agradecimentos}

À FAPESP, pelo Auxílio Pesquisa e Bolsa IC, e à Polibrasil Resinas S.A., pelo apoio, permitindo a utilização de seus equipamentos. 


\section{Referências}

1. "Lixo Municipal: Manual de Gerenciamento Integrado". Coordenação Geral: Jardim, N.S e Wells, C., IPT/CEMPRE, pp. 03-41, (1995).

2. "Meio Ambiente". Boletim Fundação Vanzolini, Ano IX, n. 42, março-abril (2000).

3. Mustafa, N. - "Plastics Waste Management: Disposal. Recycling, and Reuse". Marcel Dekker, Inc., New York, (1993).

4. "Corrida Contra o Relógio". Revista Celulose e Papel, ANFPC-UNIPRESS, Ano XIII, n. 55, agosto-setembro (1996).

5. "Números do Setor: O Balanço de 1998, Perspectivas para 1999". Revista Celulose e Papel, ANFPCUNIPRESS, Ano XV, n. 64, março-abril (1999).

6. Desai, S.C. - "Synthetic Writable Paper - a Need of the Time". Pop.Plast.Packag., v.39, n. 11, novembro (1994).

7. Huang, H.W. - "Synthetic Paper and Method for its Manufacture". Patent EP0773094A1, Publication 14/maio (1997).

8. Manrich, S. - Resultados não publicados.

9. Maruca, A.; Manrich, S. - "Caracterização de Polipropileno Reciclado de Diferentes Origens Visando a Obtenção de Papel Sintético", in: Anais do IV Congresso de Iniciação Científica-UFSCar, p.76, São Carlos - SP, 7-9, outubro (1996).
10. Fairley, M. - "Plastics, Partnerships and Profitability”. Packag.Week, v.9, n.13, setembro (1993).

11. "Synthetic Paper Offers Fast Turnaround". Paper Film Foil Conv., v. 69, n. 2, fevereiro (1995).

12. Lin, A.F. - "Process of 3-Layer Co-Extruded Biaxial Oriented Polypropylene (BOPP) Synthetic Paper". Patent US005552011A, Publication 03/setembro (1996).

13. Ohno, A. - "Synthetic Paper with Multilayer Structure and Excellent Printing Property". Patent EP0685331A1, Publication 06 dezembro (1995).

14. Takashi, H.; Takatoshi, M. - "Laminated Polyester Film”. Patent EP0795399A1, Publication 17/setembro (1997).

15. Mannar, S.M. - "Synthetic Paper and a Process for Making it". Patent EP 0605938 A1, Publication 13/julho (1994).

16. Shimada et al. - "Polyamide Solution Composition and Methods for Producing Fibrids and Paperlike Sheets Using the Same". Patent EP722987A1, Publication 24 de julho (1996).

17. Manrich, S.; Maruca, A.; Morita, A.K.M. "Caracterização de Polipropileno de Resíduo Urbano e Industrial Visando Reciclagem Mecânica Posterior", in: Anais do $4^{\circ}$ Congresso Brasileiro de Polímeros, p.805, Salvador - BA, set/out (1997).

Recebido: 03/05/00 Aprovado: 21/09/00 